\title{
Optimal Transmission Congestion Management with V2G in Smart Grid
}

\author{
Amin Niaz Azari ${ }^{1,}$,, Soodabeh Soleymani ${ }^{2}$, Babak Mozafari ${ }^{2}$, Ghazaleh Sarfi $^{3}$ \\ ${ }^{1}$ Power Distribution Company of Semnan, Semnan, Iran \\ ${ }^{2}$ Electrical Department, Islamic Azad University, Science \& Research Branch, Tehran, Iran \\ ${ }^{3}$ Electrical Department, Iran University of Science and Technology, Tehran, Iran
}

Email address:

niaamin@gmail.com(A. N. Azari), soodabeh_soleymani@yahoo.com (S. Soleymani), mozafari_babak@yahoo.com(B. Mozafari),

Gh.sarfi@gmail.com(G. Sarfi)

${ }^{*}$ Corresponding author

\section{To cite this article:}

Amin Niaz Azari, Soodabeh Soleymani, Babak Mozafari, Ghazaleh Sarfi. Optimal Transmission Congestion Management with V2G in Smart Grid. American Journal of Electrical Power and Energy Systems. Vol. 7, No. 2, 2018, pp. 16-24. doi: 10.11648/j.epes.20180702.11

Received: March 7, 2018; Accepted: March 29, 2018; Published: May 5, 2018

\begin{abstract}
The power system operators are looking for optimizing the power generating resources in the unit commitment problems considering the binding constraints. With the reconstruction in the power network structure, the increase in electricity price during some hours of day, and increase in fuel price, the utilities need to change their management paradigms. A smart grid can be a suitable choice for addressing these issues because they are able to continue working smartly. With the progress in the technology of batteries, power electronic devices, many well-known companies such as Toyota and Tesla have started producing electric and hybrid vehicles since 1990. Introducing electric vehicles to the power system provides unprecedented environmental and economic opportunities and at the same time new challenges to deal with for the system operators. The vehicle to grid (V2G) technology can enable the electric vehicles to inject energy to the grid in addition to its regular path of receiving energy from the grid. In this paper, the effect of the technology of $\mathrm{V} 2 \mathrm{G}$ on the operation cost and LMP with considering the line congestion limits are investigated. To solve the optimization problem, a mixed integer linear programming (MILP) technique in the GAMS software is used. The proposed method is tested on the IEEE 6 bus system and the results are presented. This simulation shows that although the presence of electric vehicles has no significant effect on reducing or increasing of the operation cost in smart grid and may even reduce the operation cost in a certain number of EVs, due to their daily trips and shift from a bus to another bus, they act as a transmission line during the day and reduce the line congestion, resulting in a significant reduction in the local marginal price (LMP) in the peak load hours, and also increasing the security of the power system when the line capacity falls.
\end{abstract}

Keywords: EV, Line Congestion, V2G, Unit Commitment

\section{Introduction}

Electric vehicles can be considered as loads and movable storage resources that are distributed in the entire power systems. The advent of modern power electronics has brought tremendous impact on power systems [1]. Power electronic interfaces facilitate the peneteration of renewable enrgies into the smart grids [2]. Through voltage inverters Electric Vehicles work as potential source of energy in V2G mode. The V2G technology can also make the electric vehicles to contribute to the power generation during the peak hours and decrease the operation costs. Therefore, the increase in penetration of the electric vehicles can have a significant influence on the operations of power systems. In [3], the economic benefits of electric vehicles in the ancillary services are investigated. In [4], the effects of charging PHEVs at charging stations are investigated and a strategy for improving the voltage profile and power factor in the dstribution grid is proposed. In [5], aggregators are suggested to optimally control the electric vehicles when they are connected to the network. The plug-in hybrid electric vehicles (PHEVs) are considered as regulating power providers in two case studies in Germany and Sweden [6]. In [7, 8], the technology of energy storages and power electronic components for $\mathrm{V} 2 \mathrm{G}$ 
technology have been investigated. Electric vehicles consume energy based on the distance they travel, and it is possible that the total energy that electric vehicles receive are more than they deliver to the network [9]. In [10], the incorporation of electric vehicles in a V2G mode in the Western Danish power system is investigated. In [11], the electric vehicles are considered suitable for regulatory market, spinning storage, and ancillary services but not for the based load.

Most previous studies examined only economics and technical aspects of electric vehicles and not the effects of electric vehicles with V2G technology on the operation costs and local marginal price with considering the power flow limitations in power system lines. The goal of this paper is to model the electric vehicles with V2G technology in large scale as a distributed movable load, energy storage, and power generator and their effects on optimal operation with security constraints in power systems. In this paper, the word electric vehicle (EV) is, for simplicity, used instead of the electric vehicle with V2G technology.

The rest of this paper is organized as follows. In section 2, a brief description of smart grids and the effects of electric vehicles is presented. In section 2.2, the objective function is introduced. The V2G model used in the unit commitment is introduced in section 2.3 The results and discussion are presented in section 3. Finally, the conclusion and suggestions are presented in section 4.

\section{Method}

\subsection{Smart Grid}

Nowadays, most research institutes and utilities have realized that the smart grid is a necessity for power systems. Smart grids are not new grids but the evolutionized version of current grids which aims to address the drawbacks of the exsisting networks. With incorporating advanced metering devices, e.g., phasor measurement units (PMUs) power grids have become more intelligent, reliable and efficient [12] and this give them ability to control the power system in a better way compared to traditional networks. Smart grids need to be managed actively and economically because of certain uncertainties and variability in loads and generations [14]. With the improvement in data mining, analysis, management [15], control and communication capabilities [16], smart grids aim to respond immediately to any incidents in the system [17]. Smart grids can have multiple benefits including achieving better efficiency, increasing the system reliability, incorporating more renewable energy resources, and reduced natural gas emissions [18-20].

Figure1 illustrates the presence of electric vehicles in a smart grid. As it can be seen, the independent system operator (ISO) with sending the communication signals controls the charge and discharge of electric vehicles either at home or charging stations.

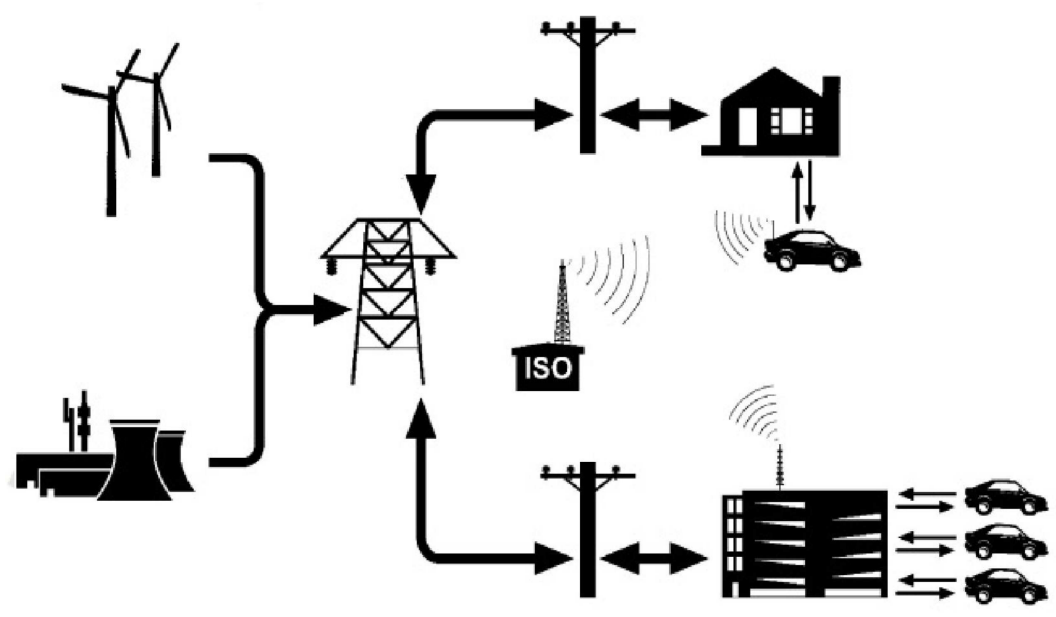

Figure 1. Two-sided communication between electric vehicles and ISO in smart grid.

\subsection{Objective Function}

In this paper, the unit commitment problem is performed by considering the limitation in the line current flows. The objective function is presented in (1).

$$
\min \left\{\begin{array}{c}
\sum_{t=1}^{T} \sum_{i=1}^{N g}\left(F_{c, i}\left(P_{i, t}\right) \cdot I_{i, t}+S U_{i, t}\right. \\
+S D_{i, t}+\sum_{t=1}^{T} \sum_{v=1}^{N v} C_{v, t}
\end{array}\right\}
$$

This objective function aims at minimizing the overall cost of generating units and calculating the optimal value for charge and discharge of EVs. The cost function of the generating unit is usually considered a polynomial function as in (2).

$$
F_{c, i}\left(P_{i}(t)\right)=a_{i} \cdot P_{i}^{2}(t)+b_{i} \cdot P_{i}(t)+c_{i}
$$

Using this equation in the optimization problem leads to a non-linear problem. A piece-wise linear model is considered for this non-linear function to linearize the function. Therefore, The new model can be presented as

$$
\begin{gathered}
F_{c, i}\left(P_{i, t}, I_{i, t}\right)=F_{c, i}\left(P_{i}^{\text {min }}\right) \cdot I_{i, t}+\sum_{k=1}^{k k} \rho_{i, t}^{k} P_{i, t}^{k} \\
0 \leq P_{i, t}^{k} \leq P_{i}^{k, \max } \forall k, \forall i, \forall t \\
P_{i, t}=P_{i}^{\text {min }} I_{i, t}+\sum_{k=1}^{k k} P_{i, t}^{k}
\end{gathered}
$$


The constraints used in this equations are 1) generation and load equality, 2) minimum and maximum of each generating unit, 3) minimum up-time of each unit, 4) minimum down-time of each unit, 5) maximum ramp-up of each unit, 6) maximum ramp-down of each unit, and 7) the limitation in the current flow of each line (security condition) [22]. In the next section, the constraints and parameters in them are explained.

\subsection{Model of V2G in Unit Commitment Problem}

To model the EVs, first, the cost function of each electrical vehicle fleets (V-fleet) is linearized, then the constrained that are used are explained. The cost function of the electrical vehicle fleet is presented as

$$
C_{v, t}=N_{v, t} \cdot\left(\sum_{m} b_{m, v} \cdot P_{m, v, t}\right) 0 \leq P_{m, v, t} \leq P_{m, v}^{\max }
$$

\section{(i) Sending and receiving energy constraint}

The net energy received by the $\operatorname{EV}\left(E_{v, t}^{n e t}\right)$, can be expressed as the difference between discharge power $\left(P_{d c, v, t}\right)$, and the effective power charged in the battery $\left(\eta_{v} \cdot P_{c, v, t}\right)$.

$$
\begin{gathered}
E_{v, t}^{n e t}=P_{d c, v, t}-\eta_{v} \cdot P_{c, v, t} \\
P_{v, t}=P_{d c, v, t}-P_{c, v, t}
\end{gathered}
$$

where $\mathrm{\eta}_{v}$ is the efficiency of the battery charging which is considered $85 \%$ in this paper.

(ii) operating modes of $E V S$

Three operating modes can be considered for the EVs, charge, discharge, and inactive.

$$
I_{d c, v, t}+I_{c, v, t}+I_{i, v, t}=N_{v, t}
$$

where $I_{d c, v, t}, I_{c, v, t}$, and $I_{i, v, t}$ denote the charge status in discharge, charge, and inactive modes of $v^{\text {th }} \mathrm{V}$-fleet at time $t$, respectively. If one of the situations above happen, $N_{v, t}$ becomes one while if it is zero it means the vechile is not connected to the grid.

(iii) Charge and discharge constraints

To charge and discharge EVs, the charge/discharge power cannot exceed or be less than certain limits.

$$
\begin{gathered}
I_{c, v, t} \cdot P_{c, v}^{\min } \leq P_{c, v, t} \leq I_{c, v, t} \cdot P_{c, v}^{\max } \\
I_{d c, v, t} \cdot P_{d c, v}^{\min } \leq P_{d c, v, t} \leq I_{d c, v, t} \cdot P_{d c, v}^{\max }
\end{gathered}
$$

(iiii) The energy of EVs batteries constraint

The energy of EVs batteries in a fleet and at a time can be express as

$$
E_{v, t}=E_{v, t-1}-E_{v, t}^{n e t}-\left(1-N_{v, t}\right) \cdot D R_{v, t}
$$

The equation above shows the energy in the batteries of $\mathrm{v}^{\text {th }}$ fleet at time $t$ equals the difference in the available energy at the previous hour with the net exchanged energy at the current time and the energy at the time the vehicle is driven.

(iiiii) Battery capacity constraint

The battery life of the EV depends heavily on the discharge level of the battery which means it must not fall below a limit. Equation (11) shows the maximum and minimum amount of the energy that can be stored in the battery of an EV.

$$
E_{v}^{\min } \leq E_{v, t} \leq E_{v}^{\max }
$$

To validate the proposed model, the simulations are performed on a six bus test system shown in Figure 2. The EVs are categorized into five different fleets based on their characteristics and daily trip pattern shown in Table 1 and Table 2, respectively [23].

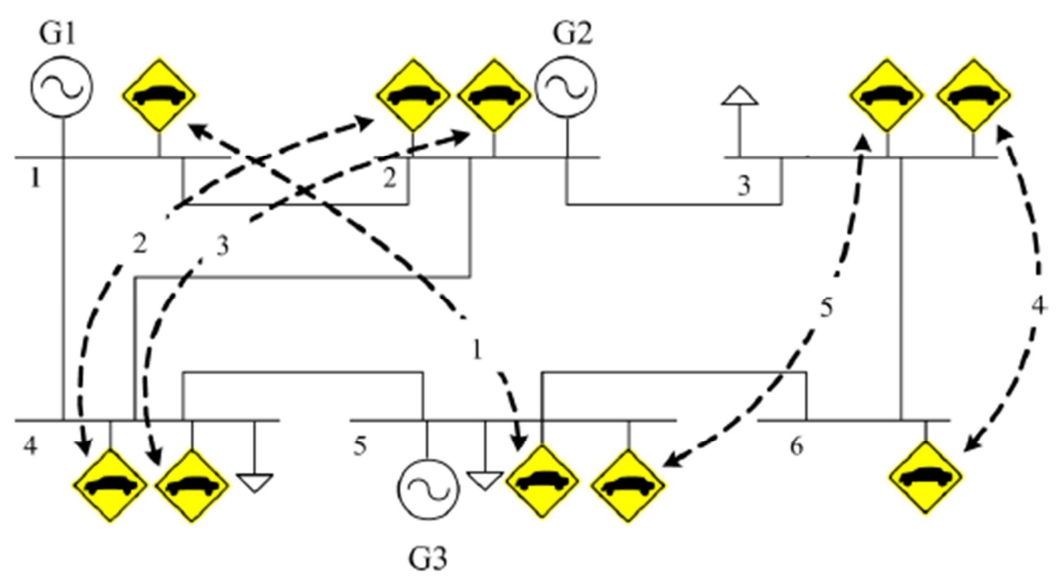

Figure 2. The six bus test system.

Table 1. Battery characteristics of EV fleet.

\begin{tabular}{llllllll}
\hline \multirow{2}{*}{ No. of Fleet } & Min. Cap (MWh) & $\begin{array}{l}\text { Max. Cap } \\
\text { (MWh) }\end{array}$ & $\begin{array}{l}\text { Min. charge/discharge } \\
\text { power }(\mathbf{k W})\end{array}$ & $\begin{array}{l}\text { Max. charge/discharge } \\
\text { power }(\mathbf{M W})\end{array}$ & $\mathbf{a}(\mathbf{\$} / \mathbf{M W})$ & $\mathbf{b}(\mathbf{\$} / \mathbf{M W})$ & $\mathbf{c}(\mathbf{\$} / \mathbf{h})$ \\
\hline 1 & 13.15 & 65.76 & $7.3 / 6.2$ & $24.8 / 21.08$ & 0.17 & 8.21 & 0 \\
2 & 10.96 & 54.8 & $7.3 / 6.2$ & $14.58 / 12.4$ & 0.20 & 8.21 & 0 \\
3 & 5.48 & 27.4 & $7.3 / 6.2$ & $7.29 / 6.2$ & 0.41 & 8.21 & 0 \\
4 & 8.768 & 43.84 & $7.3 / 6.2$ & $11.67 / 9.92$ & 0.25 & 8.21 & 0 \\
5 & 10.96 & 54.8 & $7.3 / 6.2$ & $14.58 / 12.4$ & 0.20 & 8.21 & 0 \\
\hline
\end{tabular}


Table 2. EV Fleet Trip Specifics.

\begin{tabular}{|c|c|c|c|c|c|c|c|c|c|c|}
\hline \multirow{3}{*}{ Fleet } & \multirow{3}{*}{ No. of EV } & \multicolumn{4}{|c|}{ First Trip } & \multicolumn{4}{|c|}{ Second Trip } & \multirow{3}{*}{ Energy per hr (MW) } \\
\hline & & \multicolumn{2}{|c|}{ Departure } & \multicolumn{2}{|c|}{ Arrival } & \multicolumn{2}{|c|}{ Departure } & \multicolumn{2}{|c|}{ Arrival } & \\
\hline & & Time & Bus & Time & Bus & Time & Bus & Time & Bus & \\
\hline 1 & 3400 & 6 & 5 & 8 & 1 & 17 & 1 & 19 & 5 & 7.25 \\
\hline 2 & 2000 & 7 & 4 & 8 & 2 & 16 & 2 & 17 & 4 & 9 \\
\hline 3 & 1000 & 5 & 4 & 7 & 2 & 16 & 2 & 18 & 4 & 2.25 \\
\hline 4 & 1600 & 5 & 6 & 6 & 3 & 17 & 3 & 18 & 6 & 7.2 \\
\hline 5 & 2000 & 7 & 5 & 9 & 3 & 18 & 3 & 20 & 5 & 4.5 \\
\hline
\end{tabular}

Each EV in the fleet drives 12000 miles annually and 33 miles daily on average $[24,25]$. On average, the daily consumed energy and driven distance are $9 \mathrm{~kW} /$ day and $3.65 \mathrm{miles} / \mathrm{kWh}$, respectively [26]. In this paper, it is assumed that the consumed energy in the departure and arrival routes are the same.

To solve the unit commitment problem, the mixed-integer linear programming (MILP) technique is deployed, and the simulations are performed in GAMS software which is a very powerful tool for dealing with optimization problems. To solve the problem, the CPLEX method which is one of the efficient techniques for solving the MIP is used [27].

\section{Result and Discussion}

Using the mentioned method, the problem is solved in three modes and. 1) without considering of the EVs, 2) with considering the EVs but assuming they are not moving, and 3) with considering the EVs and assuming they are moving. The results are shown in Table 3. As it can be seen the operation cost for the third case is $\$ 134345$.

Table 3. Operating cost comparison.

\begin{tabular}{ll}
\hline Without EVs & $\$ 136335$ \\
With EVs and not moving & $\$ 130869$ \\
With EVs and moving & $\$ 134345$ \\
\hline
\end{tabular}

Table 4 shows the charge and discharge of EV fleet during 24 hours. It is noticeable from the table that during hours 1 and 12 where there is no peak load, the batteries are charged. On the other hand, during hours 13 and 24 where the network faces the peak load, most of the fleets are in the discharge mode (the negative power means charging, and positive power means discharging). If it is assumed that the EVs are not moving during the day and are always connected to grid in the charging parking, then the operation cost reduces to $\$ 130869$. This is because in this case no energy is consumed for driving the EV. Table 3 shows that the presence of EVs in the grid reduces the operation costs.

Table 4. The amount of charge and discharge power of the EV fleet per hour in terms of megawatt in 24 hours.

\begin{tabular}{|c|c|c|c|c|c|c|}
\hline \multirow{3}{*}{ Fleet } & \multicolumn{6}{|c|}{ Hours between (1-12) } \\
\hline & 1 & 2 & 3 & 4 & 5 & 6 \\
\hline & 7 & 8 & 9 & 10 & 11 & 12 \\
\hline \multirow{2}{*}{1} & 0 & 0 & 0 & -8.27 & -9.72 & 0 \\
\hline & 0 & -9.72 & 0 & -3.55 & -9.72 & -9.72 \\
\hline \multirow{2}{*}{2} & 0 & 0 & 0 & 0 & 0 & 0 \\
\hline & 0 & 0 & 0 & 0 & -5.71 & -11.1 \\
\hline 3 & 0 & 0 & 0 & 0 & 0 & 0 \\
\hline \multirow{2}{*}{4} & -2.49 & -4.98 & -8.97 & -9.14 & 0 & -6.67 \\
\hline & -9.14 & -4.57 & -3.74 & 0 & 0 & 0 \\
\hline \multirow{2}{*}{5} & -5.71 & -5.71 & -8.87 & -8.41 & -11.43 & -11.43 \\
\hline & 0 & 0 & -5.71 & -3.98 & 0 & 3.05 \\
\hline \multirow{3}{*}{ Fleet } & Hours & & & & & \\
\hline & 13 & 14 & 15 & 16 & 17 & 18 \\
\hline & 19 & 20 & 21 & 22 & 23 & 24 \\
\hline \multirow{2}{*}{2} & -8.21 & -10.24 & -11.43 & 0 & 0 & 0 \\
\hline & 4.13 & 4.13 & 4.13 & 1.42 & 4.13 & 0 \\
\hline \multirow{2}{*}{3} & -5.11 & -2.85 & -11.43 & 0 & 0 & 0 \\
\hline & 2.06 & 2.06 & 2.06 & 0 & 0 & 0 \\
\hline \multirow{2}{*}{4} & 0 & 0 & 0 & 0 & 0 & 3.30 \\
\hline & 8.22 & 6.60 & 4.43 & 1.99 & 3.30 & 0 \\
\hline \multirow{2}{*}{5} & 4.74 & 4.32 & 1.77 & 2.65 & 4.21 & 0 \\
\hline & 0 & 5.14 & 4.13 & 3.06 & 0.97 & 0 \\
\hline
\end{tabular}

With the closer look at the charging and discharging process at Table 4, it can be seen that when the EVs are located at bus 6 and then move to bus 3 at 5 o'clock, they are charging between hours 1 and 9 (except hour 5), which is due to the low local marginal price at these buses during these hours. In addition, it can be seen from Figure 3 that bus 6 has the highest of its local marginal price during the hours 18 and 24 . Therefore, when the EVs of fleet 4 go this bus, they give energy to the grid 
during these hours. Moreover, Figure 3 shows that bus 3 and bus 2 have the maximum, and minimum amount of local marginal price (LMP) during hours 10 and 17 . Consequently according to tables 2 and 4 when EVs are connected to the grid at bus 3 (fleets 4 and 5) during these hours, they are not charging and prefer to sell power to the grid and when EVs are connected to bus 2 (fleets 2 and 3), they do not give energy to the grid.

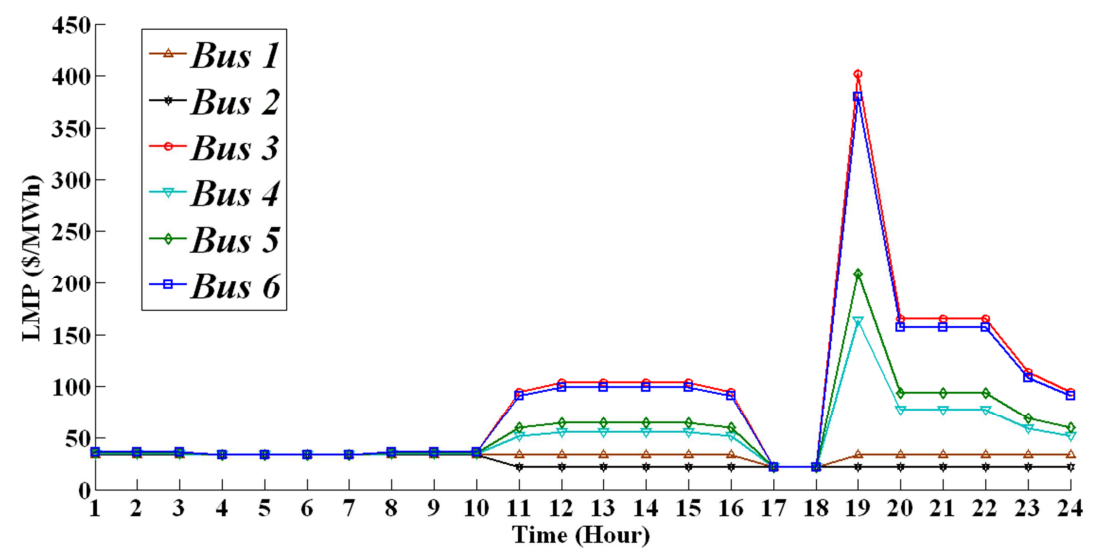

Figure 3. LMP of the network without the presence of EVS.

Figure 4 shows that with the presence of EVs in the network and considering that they are moving, during hours 18 and 24 which are the peak load hours, the LMP decreases significantly.

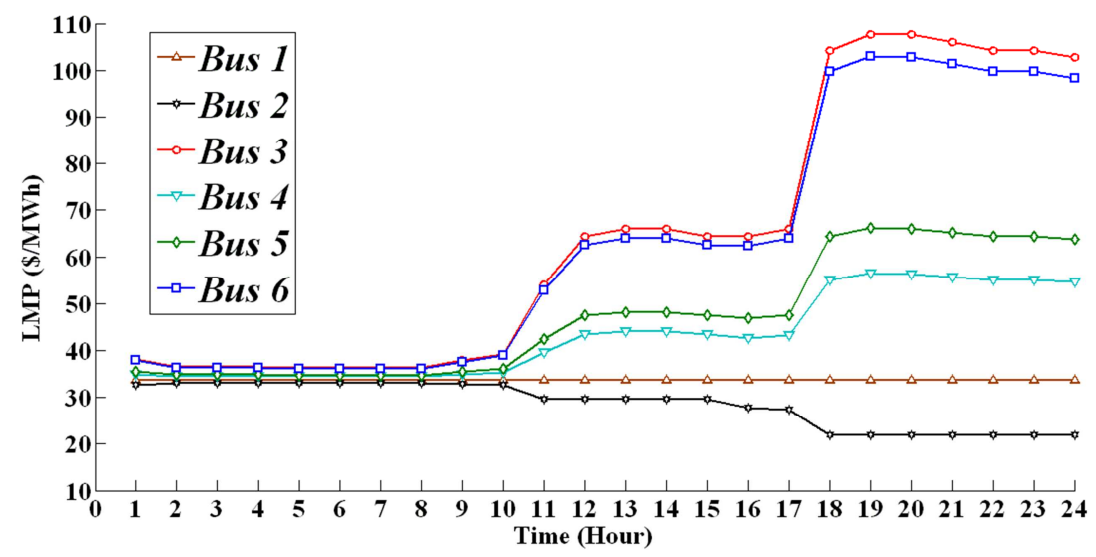

Figure 4. LMP of the network considering the presence of moving EVs.

Figure 5 shows the LMP of the network with the presence of EVs and considering that they are not moving. This figure shows that LMPs of buses 3, 4, 5, and six from hour 10 have reached their maximum values. While according to Figure 4 from hour 18 the LMP at this buses have reached their maximum values. This obviously shows that moving the EVs in the grid reduces line congestion and consequently reduces the LMPs of these buses from hours 10 and 18.

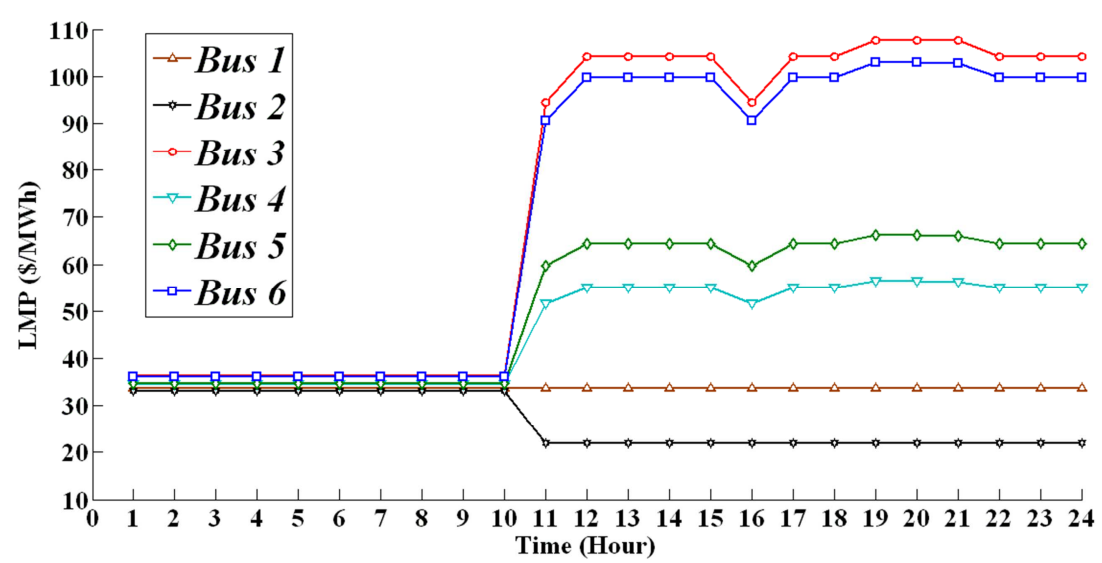

Figure 5. LMP of the network considering the presence of not moving EVS. 
To investigate the effect of the line congestion on the system security, we decrease the capacity factor of line 3 . As it can be seen from Figure 6. When the EV fleet is not in the network, the network can maintain stable only 0.7 of the line 3 capacity factor. While, when the EVs are present in the network, they can maintain the system security up to 0.3 of line 3 capacity factor and when the capacity of the line 3 is reduced to 0.3 , just the movement of EVs can provide system security. Moreover when EVs are present in the network while the congestion of lines increases, the movement of EVs creats less operation cost compared with they are not moving.

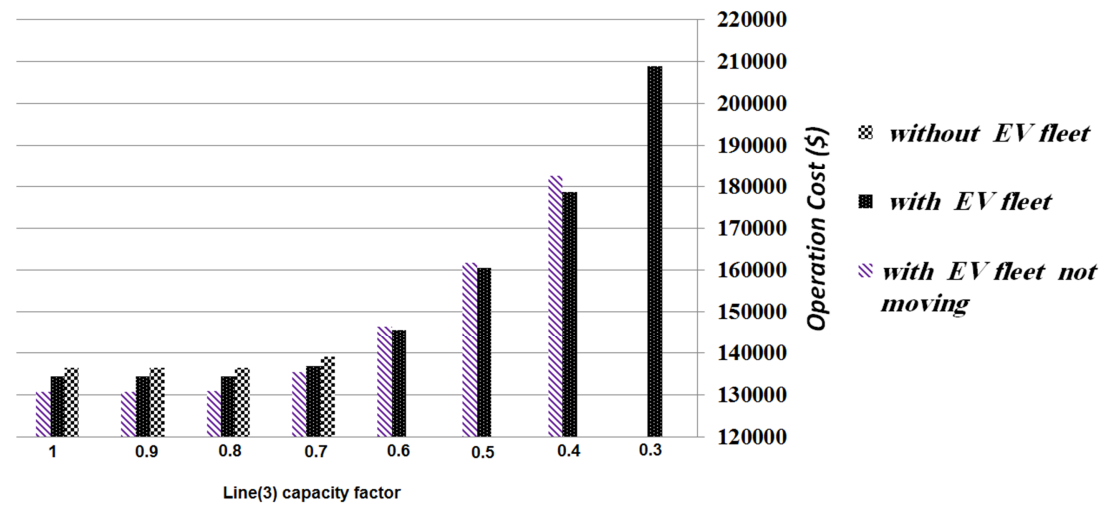

Figure 6. System security with an increase in the line congestion.

Figure 7 shows the sensitivity of the operation cost concerning the number of EVs. It can be seen from the figure that if the number of EVs become 0.7 of the anticipated number, then the operation cost becomes minimum. Also, with the increase in the number EVs from 0.2 to 0.8 of the current number, the operation cost of the thermal unit decreases. However, after 0.8 , the operation cost increases. This is because, with the increase in the number of EVs, the charge/discharge costs of batteries, and therefore, the power generation cost of the thermal units increase. Moreover, this figure shows that with the increase in the number of the EVs, the operation cost of EVs increase. However, after 0.8 the curve tends to become a constant. This is because with the increase in the number of EVs the charge/discharge cost of batteries increase and therefore, the operator prefers to use the thermal units instead of using the extra power provided by EVs.

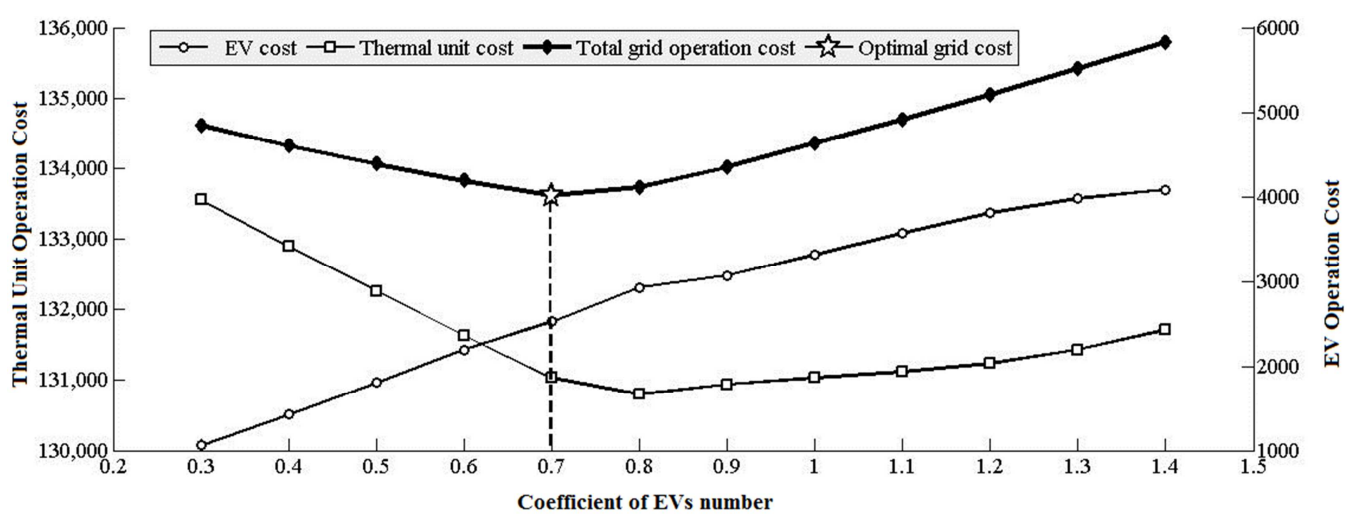

Figure 7. The sensitivity of the operation cost concerning the number of EVS.

Table 5. Status Of Charging (SOC) of the EV fleets in 24 hours in percent.

\begin{tabular}{|c|c|c|c|c|c|c|}
\hline \multirow{3}{*}{ Fleet } & \multicolumn{6}{|c|}{ Hours between (1-12) } \\
\hline & 1 & 2 & 3 & 4 & 5 & 6 \\
\hline & 7 & 8 & 9 & 10 & 11 & 12 \\
\hline \multirow{2}{*}{1} & 20 & 20 & 20 & 30.70 & 43.27 & 31.63 \\
\hline & 20 & 32.57 & 32.57 & 37.16 & 49.73 & 62.30 \\
\hline \multirow{2}{*}{2} & 36.42 & 36.42 & 36.42 & 36.42 & 36.42 & 36.42 \\
\hline & 20 & 20 & 20 & 20 & 28.86 & 46.18 \\
\hline \multirow{2}{*}{3} & 36.42 & 36.42 & 36.42 & 36.42 & 28.21 & 20 \\
\hline & 20 & 20 & 20 & 20 & 32.21 & 41.07 \\
\hline \multirow{2}{*}{4} & 24.84 & 34.50 & 51.90 & 69.64 & 53.21 & 66.15 \\
\hline & 83.88 & 92.75 & 100 & 100 & 100 & 100 \\
\hline \multirow{2}{*}{5} & 28.86 & 37.73 & 51.49 & 64.54 & 82.27 & 100 \\
\hline & 91.79 & 83.58 & 92.44 & 98.63 & 98.63 & 93.05 \\
\hline
\end{tabular}




\begin{tabular}{|c|c|c|c|c|c|c|}
\hline \multirow{3}{*}{ Fleet } & \multicolumn{6}{|c|}{ Hours between (1-12) } \\
\hline & 1 & 2 & 3 & 4 & 5 & 6 \\
\hline & 7 & 8 & 9 & 10 & 11 & 12 \\
\hline & \multicolumn{6}{|c|}{ Hours between (13-24) } \\
\hline \multirow[t]{2}{*}{ Fleet } & 13 & 14 & 15 & 16 & 17 & 18 \\
\hline & 19 & 20 & 21 & 22 & 23 & 24 \\
\hline \multirow{2}{*}{1} & 74.78 & 74.78 & 87.43 & 100 & 88.37 & 76.73 \\
\hline & 64.31 & 53.63 & 42.95 & 32.27 & 21.58 & 20 \\
\hline \multirow{2}{*}{2} & 58.91 & 75.42 & 93.15 & 76.42 & 76.72 & 69.18 \\
\hline & 61.65 & 54.11 & 46.57 & 43.96 & 36.42 & 36.42 \\
\hline \multirow{2}{*}{3} & 56.93 & 65.79 & 75.45 & 67.24 & 59.03 & 59.03 \\
\hline & 51.49 & 43.96 & 36.42 & 36.42 & 36.42 & 36.42 \\
\hline \multirow{2}{*}{4} & 100 & 100 & 100 & 100 & 83.58 & 76.04 \\
\hline & 57.27 & 42.20 & 32.09 & 27.54 & 20 & 20 \\
\hline \multirow{2}{*}{5} & 84.38 & 76.50 & 73.26 & 68.41 & 60.72 & 52.51 \\
\hline & 44.30 & 34.90 & 27.36 & 21.77 & 20 & 20 \\
\hline
\end{tabular}

Table 5 shows that how the smart grid controls status of charging (SOC) of the EVs battery in 24 hours, in order to operate the network at a minimum cost, and also provide ample energy for the EVs in daily trips.

\section{Conclusion}

In this paper, the unit commitment problem with considering the electric vehicles (EVs) with V2G technology and security constraints is solved. In this paper, the EVs are modeled in large scale in a smart grid. The model is linearized and is implemented with MIP in the GAMS software and is solved with CPLEX method. The effects of the EVs on the operation cost and power generation of thermal units are investigated. Furthermore, the impacts of displacing the EVs on the system security and local marginal price are studied. The results show that with the presence of the EVs in the peak loads hours and injection of the power to grid, there is no need for expensive units to generate power. The moving ability of the EVs, makes them to serve as the transmission lines and transmit power from one bus to another and reducing the line congestion and consequently, decreasing the LMP. Furthermore, due to the energy saving ability of the EVs and generating power, the system security increases.

\section{Nomenclaure}

Cost function of $i^{\text {th }}$ unit

Power generation of $i^{\text {th }}$ unit at time $t$

charge power / discharge power of $\mathrm{v}^{\text {th }}$ fleet at time $\mathrm{t}$

The operating cost of electrical vehicle fleets

The available energy in the batteries of $v^{\text {th }}$ fleet at time $t$

Min / Max energy stored in the battery of the $\mathrm{v}^{\text {th }}$ fleet

Minimum / Maximum charge rate of the $v^{\text {th }}$ fleet

Minimum / Maximum discharge rate of the $\mathrm{v}^{\text {th }}$ fleet

Charging mode of $\mathrm{v}^{\text {th }}$ fleet

Discharging mode of $\mathrm{v}^{\text {th }}$ fleet

Idle mode of $\mathrm{v}^{\text {th }}$ fleet

On / Off mode of $i^{\text {th }}$ unit at time $t$

Total load of network

Total number of buses

The number of thermal units located on the $b^{\text {th }}$

bus

The total number of transmission lines

The slope of the cost function for k-piece

the slope of the $\mathrm{m}$-part of the linear charging and discharging curve of the $\mathrm{v}^{\text {th }}$ fleet

the linear charging and discharge curve of the vth fleet at time $t$ and in mth part

Net energy delivered to the network

The number of transmission lines located at the $b^{\text {th }}$ bus

The amount of energy consumed by each fleet per hour of movement

The minimum output power of $i^{\text {th }}$ unit

Maximum output power of $i^{\text {th }}$ unit
$F_{c, i}($.

$P_{i, t}$

$P_{d c, v, t} / P_{c, v, t}$

$C_{(.)}^{(.)}$

$E_{v, t}^{(.)}$

$E_{v}^{\max } / E_{v}^{\min }$

$P_{c, v}^{\max } / P_{c, v}^{\min }$

$P_{d c, v}^{\max } / P_{d c, v}^{\min }$

$I_{c, v, t}$

$I_{d c, v, t}$

$I_{i, v, t}$

$I_{i, t}$

$P_{D,[.)}^{(.)}$

$N B$

$N G$

$b$

$N L$

$\rho_{i, t}^{k}$

$b_{m, v}$

$P_{m, v, t}$

$E_{v, t}^{n e t}$

$N L_{b}$

$D R_{v, t}$

$P_{i}^{\text {min }}$

$P_{i}^{\max }$ 
The startup cost of $i^{\text {th }}$ unit at t-time

The shutdown cost of the $i^{\text {th }}$ unit at time $t$

The reactance of line 1

The angle of each bus

The maximum transmission capacity of line 1

The state of connection to the network of $v^{\text {th }}$ fleet at time $t$

Charging cycle efficiency of the $\mathrm{v}^{\text {th }}$ fleet

$S U_{i, t}$
$S D_{i, t}$
$X_{l}$
$\theta_{(.)}$
$P L_{l}^{\max }$
$N_{v, t}$
$\eta_{v}$

[13] M. A. Chitsazan, A. M Trzynadlowski, "State estimation of IEEE 14 bus with interphase power controller using WLS method", Energy Conversion Congress and Exposition (ECCE), 2016 IEEE, pp. 1-5, Sep. 2016.

[14] V. Sarfi, I. Niazazari, and H. Livani, "Multiobjective fireworks optimization framework for economic emission dispatch in microgrids." North American Power Symposium (NAPS), 2016, pp. 1-6, Nov. 2016.

[15] A Ghasemkhani, L Yang, "Reinforcement Learning based Pricing for Demand Response," in Communications Workshops (ICC Workshops), 2018 IEEE International Conference on. IEEE, 2018.

[16] A. Ghasemkhani, A. Anvari-Moghaddam, J. M. Guerrero, and B. Bak-Jensen, "An Efficient Multi-objective Approach for Designing of Communication Interfaces in Smart Grids," Proceedings of IEEE PES Innovative Smart Grid Technologies, Ljubljana, Slovenia (ISGT Europe 2016), Oct. 2016.

[17] A. Forooghi Nematollahi, A. Dadkhah, O. Asgari Gashteroodkhani, and B. Vahidi, "Optimal sizing and siting of DGs for loss reduction using an iterative-analytical method," Journal of Renewable and Sustainable Energy, vol. 8, no. 5, p. 055301, 2016.

[18] V. Sarfi and H. Livani, "A novel multi-objective security-constrained power management for isolated microgrids in all-electric ships," 2017 IEEE Electric Ship Technologies Symposium (ESTS), 2017.

[19] O. A. Gashteroodkhani, B. Vahidi, and A. Zaboli, "Time-time matrix Z-score vector-based fault analysis method for series-compensated transmission lines," Turkish Journal of Electrical Engineering \& Computer Sciences, vol. 25, pp. 2647-2659, 2017.

[20] V. Sarfi, H. Livani, and L. Yliniemi, "A Novel Multi-Objective Economic-Emission Dispatch in Microgrids" IEEE Power Engineering Society General Meeting, Chicago, Illinios, July 2017.

[21] M. A. Chitsazan, M. Sami Fadali, Amanda K. Nelson, A. M Trzynadlowski, "Wind speed forecasting using an echo state network with nonlinear output functions", American Control Conference (ACC), 2017 IEEE, pp. 5306-5311, May. 2017.

[22] J .Wang, M. Shahidehpour, Z .Li, "Security Constrained Unit Commitment With Volatile Wind Power Generation", IEEE Transactions on Power Systems, vol. 23, NO. 3, August 2008.

[23] M. Khodayar and M. Shahidehpour, "Hourly Coordination of Electric Vehicle Operation and Volatile Wind Power Generation in SCUC" IEEE Transactions on Smart Grid, Vol. 3, No. 3, September 2012.

[24] A. Saber and G. Venayagamoorthy, "Plug-in vehicles and renewable energy sources for cost and emission reduction," IEEE Trans. Ind. Electron, 2010. 
[25] C. Roe, A. P. Meliopoulos, J. Meisel, and T. Overbye, "Power system level impacts of plug-in hybrid electric vehicles using simulation data,"in Proc. IEEE Energy 2030, Atlanta, GA, Nov. 17-18, 2008.

[26] J. Tomic and W. Kempton, "Using fleets of electric-drive vehicles for grid support," J. Power Sources, vol. 168, no. 2, pp. 459-468, Jun. 2007.
[27] GAMS Documentation [Online]. Available: http://www.gams.com/docs/document.htm. 